\title{
Estudio Piloto: Descripción de la Carga Global de Trabajo, el Factor Físico-Biomecánico y Percepción de Molestias Músculo-Esqueléticas en Trabajadoras Embarazadas
}

\section{PILOT STUDY: DESCRIPTION OF GLOBAL WORKLOAD, BIOMECHANICAL FACTOR AND PERCEPTION OF MUS- CULOSKELETAL DISCOMFORT IN PREGNANT WORKERS}

\author{
Carolina Rodríguez Herrera', Eduardo Cerda Díaz', Jorge Rodríguez Tobar², Carlos Díaz Canepaㄹ, Álvaro Besoain Saldaña', \\ Giovanni Olivares Péndola', Rodrigo Pinto Retamal', Lorena Araos Bravo', Myria Arévalos Valdivia' \\ 1. Laboratorio de Ergonomía, Departamento de Kinesiología, Facultad de Medicina, Universidad de Chile, Santiago, Chile. \\ 2. Escuela de Salud Pública, División de Bioestadística, Facultad de Medicina, Universidad de Chile, Santiago, Chile. \\ 3. Facultad de Ciencias Sociales. Universidad de Chile, Santiago, Chile. \\ 4. Asociación Chilena de Seguridad, Santiago, Chile.
}

\begin{abstract}
RESUMEN
Se ha identificado una sobrecarga laboral en mujeres durante el embarazo. Suelen presentar una alta prevalencia de trastornos músculo-esqueléticos, no obstante existe poca evidencia sobre su progresión en los trimestres y asociación con carga de trabajo. Objetivo: Describir la carga de trabajo, factor físico biomecánico y percepción de molestias músculo-esqueléticas (PMME) en trabajadoras embarazadas. Material y método: Estudio de diseño observacional, descriptivo y transversal. Muestreo por conveniencia de 80 mujeres embarazadas de la Unidad de Medicina Materno Fetal del Hospital Clínico de la Universidad de Chile. Se describe la muestra a través de la evaluación de carga de trabajo, factores físicos biomecánicos, dificultades percibidas al realizar su tarea mediante una hoja de recolección de información diseñada específicamente para la investigación, usando metodología en ergonomía Resultados: Las PMME presentan una prevalencia de un 90\%, destacando la zona de la espalda baja y cadera/nalgas/muslos. Las posturas mantenidas o forzadas fueron el factor más prevalente. Una de cada 6 embarazadas refirieron continuar realizando manejo manual de carga. La mayoría de las trabajadoras presentaron un alto riesgo en su carga de trabajo. Conclusiones principales: Las embarazadas están expuestas a altos niveles de carga de trabajo determinado por exigencia mental.
\end{abstract}

(Rodríguez C, Cerda E, Rodríguez J, Díaz C, Besoain A, Olivares G, Pinto R, Araos L, Arévalos M, 2017. Estudio Piloto: Descripción de la Carga Global de Trabajo, el Factor Físico-Biomecánico y Percepción de Molestias Músculo-Esqueléticos en Trabajadoras Embarazadas. Cienc Trab. Ene-Abr; 19 [58]: 1-6).

Palabras claves: MUJERES EMBARAZADAS, CARGA DE TRABAJO, TRABAJO DE MUJERES, INGENIERÍA HUMANA.

\section{ABSTRACT}

It have been detected overload work among women during pregnancy. Often they present a high prevalence on HYPERLINK "https:// www.google.cl/search?q=work+related+musculoskeletal+disorder\&s pell=1\&sa $=$ X\&ved=0ahUKEwiw2IS2gePTAhWDD5AKHbK5DcoQvw UIIigA" work related musculoskeletal disorder; however there is little evidence about their progression during the pregnancy's trimester and the association with work load. Objective: Describe work load, physical and biomechanical factors, perception of musculoskeletal discomfort (PMSD) in pregnant women. Materials and methods: Observational, descriptive and cross-sectional study. Sampling by convenience of 80 pregnant women of the Maternal-fetal care unit of the Clinical Hospital of the University of Chile. The sample is described trough the evaluation of workload, physical and biomechanical factors, trouble performing tasks by means of a sheet of collection of information created for this investigation, using ergonomics methodology. Results: The PMSD present a prevalence of $90 \%$, stands out the lower back and hips/buttocks/thigh areas. The forced or maintained postures ware the most prevalent factor. One in six pregnant women referred continuing performing weight manual handling tasks. Most of the women presents high workload. Main conclusion: Pregnant women are exposed to high levels of workload determined by mental exigence.

Key Words: PREGNANT WOMAN, WORKLOAD, WOMEN'S WORK, HUMAN ENGINEERING
Correspondencia / Correspondence:

Dr. Erg. Eduardo Cerda Díaz

Laboratorio de Ergonomía, Departamento de Kinesiología,

Facultad de Medicina, Universidad de Chile.

Av. Independencia \# 1027; Santiago de Chile.

Tel. +5622978651

e-mail: encerda@med.uchile.cl

Recibido: 19 enero 2017 / Aceptado: 12 de febrero de 2017

\section{INTRODUCCIÓN}

Tanto a nivel internacional como nacional la inserción de la mujer en el mundo laboral es cada vez mayor, y se estima que esta seguirá en aumento. ${ }^{1}$ En Chile, la tasa de ocupación de la mujer del trimestre diciembre-febrero 2010 es del 37,8\%. En relación a la distribución porcentual de la ocupación: "El sector primario es el tercero en importancia porcentual de la ocupación femenina, tras el sector terciario $(85,3 \%)$ y el sector secundario $(9,8 \%))^{2}$. A su vez, se ha evidenciado un patrón de exposición de factores de riesgo en el lugar 
de trabajo diferenciado para hombres y mujeres, dado las diferencias de género.

En cuanto al nivel de participación de la mujer en el trabajo se hace crucial ante el cambio de la planificación familiar y ciclo de vida de la mujer, ya que según el Instituto Nacional de Estadísticas el número de nacimientos de madres laboralmente activas alcanza el 45\% de los nacimientos totales. ${ }^{3}$ Por ello, se ha identificado un aumento del número de mujeres trabajadoras que pueden enfrentarse al factores de riesgo ergonómicos en su periodo de gestación.

Otro factor relevante en el contexto de la investigación y el desarrollo de políticas en salud del trabajo es el hecho de que muchas veces se ha planteado que el trabajo que realizan las mujeres no suele presentar riesgos para su salud; esto lleva a una disminución de la investigación en este tema y a una falta de información sobre los riesgos a los cuales están expuestas las mujeres. ${ }^{4}$ De hecho, según la Encuesta de Calidad de vida y Salud (2007), la conciliación de la vida familiar y la vida laboral afecta primordialmente a las trabajadoras, lo cual se evidencia a través de una doble carga percibida en el trabajo. ${ }^{5}$

"En el ámbito laboral, la exigencia de trabajo por lo general se mantiene; sin embargo, la capacidad de la embarazada para responder a dicha exigencia dependerá de su capacidad, pudiendo esta aumentar o disminuir según la evolución de sus cambios fisiológicos”6, lo que hace fundamental considerar a las mujeres no sólo en el contexto de las actividades de la vida diaria con sus diversos cambios en los componentes físicos y psicosociales, sino también en las mujeres en situación de embarazo que se encuentran en el ámbito laboral.

Respecto al punto anterior, distintas publicaciones han establecido la necesidad de realizar intervenciones en los puestos de trabajo de embarazadas. ${ }^{7-10}$ Los principales ejes a intervenir son: a) La presencia de factores de riesgo de Trastornos Músculo-Esqueléticos Relacionados con el Trabajo ${ }^{11-15}$; b) Disminuir los factores de riesgo ergonómicos presentes en puestos de trabajo de embarazadas ${ }^{16,17}$; c) Disminuir la carga cognitiva de los puestos y jornadas de trabajo ${ }^{18-21}$; d) Controlar los factores psicosociales ${ }^{21}$, entre otros. Esto es sustantivo con la existencia de distintos estudios sobre intervenciones que han demostrado tener impacto en la sobrecarga física y mental de la mujer en sus puestos de trabajo. ${ }^{7-10,21-24}$

El desarrollo de una identificación de los factores de riesgo de la salud y calidad de vida de embarazadas permite reducir una sobrecarga de la mujer, poniendo en riesgo su desarrollo tanto laboral como en su embarazo. ${ }^{25-28}$ Por lo anterior, es necesario contextualizar desde el punto de vista del factor físico-biomecánico a que se encuentran sometidas las trabajadoras embarazadas, a través de la descripción de los mismos, determinar las dimensiones de la carga global de trabajo, presencia de factores físico-ergonómicos y la percepción de molestias músculo-esqueléticas de trabajadoras en situación de embarazo, que puedan servir de base para determinar recomendaciones iniciales que ayuden en el autocuidado de las embarazadas.

\section{MATERIAL Y MÉTODO}

Se realizó un estudio de diseño observacional, de tipo descriptivo $\mathrm{y}$ transversal. Con un muestreo por conveniencia, comprendiendo 80 mujeres embarazadas entre las semanas 7 y 34 de gestación, con una jornada laboral de al menos 22 horas, quienes se atendían en la Unidad de Medicina Materno Fetal del Hospital Clínico de la Universidad de Chile. Se excluyeron las mujeres embarazadas que estuvieran siendo atendidas por un cuadro médico de urgencia; con embarazos múltiples; complicaciones del embarazo asociadas a hipertensión, diabetes mellitus, trastornos psicológicos (depresión endógena) o con tratamiento farmacológico por existencia de patología de base.

El desarrollo de esta investigación cuenta con la aprobación del Comité de Ética del Hospital Clínico de la Universidad de Chile, Santiago, Chile, y presentación al Director del Departamento de Obstetricia y Ginecología del Hospital Clínico de la Universidad de Chile.

Para la recolección de datos, primero se realizó un proceso de consentimiento informado, el cual contaba con una exposición de las características del estudio y consideraciones relevantes del estudio. Tras resolver cualquier duda que hubiesen tenido, se procedió a realizar la firma del consentimiento informado. Una vez realizado esto, se aplicó una hoja de recolección de información considerando variables sociodemográficas, gineco-obstétricas y de salud a través de la medición de altura y peso y el análisis del IMC propuesto por Atalah. ${ }^{29} \mathrm{El}$ protocolo es descrito en la Figura 1. La evaluación de los factores ergonómicos físicos y mentales suelen caracterizarse como herramientas de terreno basadas principalmente en el análisis observacional. Sin embargo, involucrar la percepción de los trabajadores en cuestionarios de autorreporte se ha vuelto un pilar que permite complementar el análisis de las interacciones del sistema de trabajo y la persona. ${ }^{30,31}$

Figura 1.

Esquema sobre el reclutamiento y protocolo de recolección de datos.

$\left.\left.\sum \begin{array}{c}\text { Reclutamiento } \\ (\text { HCUCH) }\end{array}>\sum \begin{array}{c}\text { Consentimiento } \\ \text { informado } \\ \text { (Sala privada) }\end{array}>\sum \begin{array}{l}\text { Análisis de } \\ \text { sistema } \\ \text { (Entrevista) }\end{array}\right\rangle \sum \begin{array}{c}\text { Recolección } \\ \text { de datos } \\ \text { (Cuestionario) }\end{array}\right\rangle$

Adicionalmente, se utilizaron distintos instrumentos autoaplicados y lista de chequeo sobre factores de riesgo laborales, con tal de describir de manera integral la condición de salud músculo-esquelética y carga global de trabajo de las mujeres embarazadas. Se indagaron las distintas variables: a) Factor Físico-Biomecánico, en base a un ítem de la lista de chequeo de la "Guía Técnica para la evaluación del trabajo pesado". ${ }^{32}$ Esta lista de chequeo busca sistematizar la identificación de factores de riesgo ergonómico. Los factores físicos se detectan en base a los datos proporcionados por la trabajadora a través de la información del proceso de trabajo, que relata de su jornada laboral; b) Percepción de molestias músculo-esqueléticas, a través del Cuestionario Nórdico Estandarizado para detectar síntomas músculo-esqueléticos, validado en Chile $^{33}$. Este instrumento evalúa en nueve regiones corporales: cuello, los hombros, la parte superior espalda, los codos, la zona lumbar, muñeca/manos, caderas/muslos, las rodillas y tobillos/ pies; c) Carga global de trabajo percibida a través del método NASA-TLX. Este instrumento consta de preguntas auto-administradas sobre el impacto del trabajo en dimensiones mentales y físicas. Cada dimensión presenta un impacto de 0 a 100, el cual es ponderado según la perspectiva de la persona. La suma del puntaje final ponderado por dimensión entrega el Puntaje Ponderado Total (PPT), el cual puede ser clasificado en Carga leve (0 a 500 puntos), carga moderada (501 a 1000 puntos) y carga alta (1001 a 1500 puntos) $(29,30,32)$; d) Dificultades percibidas por la trabajadora embarazada al realizar su tarea o actividad laboral, basadas en las 
principales dificultades en los puestos de trabajo, descritas en el Ergomater IBV $^{34}$ y estudios científicos ${ }^{35}$, describiendo una lista de 13 dificultades.

\section{Análisis estadístico}

La información recolectada desde los instrumentos fue procesada para el posterior análisis de datos; para las variables cualitativas, se utilizaron frecuencias absolutas y distribuciones porcentuales. Se utilizó la prueba de Shapiro \& Wilks para identificar el tipo de distribución de los datos. Se utilizaron promedios y desviaciones estándares si los datos distribuian normal, y medianas y rangos intercuartílico si no distribuían normal. Para el cruce entre variables cualitativas se utilizó la prueba de ji cuadrado. Para la prueba de hipótesis de variables que distribuian normal, se utilizó la prueba de t de student para muestras independientes para comparar entre parejas, y la prueba de ANOVA con la corrección de Bonferroni como método post-hoc. En las variables sin distribución normal, se utilizó la prueba de Mann-Whitney para comparar dos grupos, y la prueba de Kurskal-Wallis para más de 2 grupos, con la prueba de Bernard-Rosner como método post-hoc. Para el procesamiento de datos y análisis estadístico, se utilizó el programa IBM SPSS Statistics for Windows, Version 19.0. Armonk, NY: IBM Corp. para el análisis de datos.

\section{RESULTADOS}

Se obtuvo una muestra de 80 mujeres, con una mediana de 31 años, principalmente del segundo trimestre de gestación. En la Tabla 1 se describen las características sociodemográfica de la muestra obtenida. En el Tabla 2 se describe la prevalencia de molestias músculo-esqueléticas (MME) en embarazadas, siendo estas altamente frecuentes, con una distribución porcentual de $90 \%$ para la población total, dentro de lo cual las zonas corporales que presentaban mayor prevalencia fueron espalda baja $(77,5 \%)$, cadera/nalgas/muslos $(42,5 \%)$, tobillos/pies $(26,3 \%) \mathrm{y}$ espalda alta (25\%).

Tabla 1.

Descripción sociodemográfica y gineco-obstétrica de la muestra. Se entrega mediana (01-03).

\begin{tabular}{lcc}
\multicolumn{1}{c}{ Variable } & Valor \\
Edad (años) & & $31(27,5-35)^{*}$ \\
Tiempo de Gestación (semanas) & & $22(13-29)^{*}$ \\
Peso $(\mathrm{kg})$ & & $70,9(62,8-78)^{*}$ \\
Altura $(\mathrm{m})$ & & $1,6(1,55-1,63)^{*}$ \\
IMC según Atalah $\mathrm{n}(\%)$ & Bajo peso & $3(3,8)$ \\
& Normopeso & $27(33,8)$ \\
& Sobrepeso & $33(41,2)$ \\
Estado Civil $\mathrm{n}(\%)$ & Obesidad & $17(21,2)$ \\
& Soltera & $53(66,2)$ \\
Número de Embarazos $\mathrm{n}(\%)$ & Casada & $24(30)$ \\
& Separada & $3(3,8)$ \\
& Primigesta & $25(31,3)$ \\
Número de partos $\mathrm{n}(\%)$ & 2 embarazos & $35(43,7)$ \\
& 3 o más embarazos & $20(25)$ \\
& Nulipara & $34(42,4)$ \\
& 1 parto & $31(38,8)$ \\
& 2 partos & $11(13,8)$ \\
& 3 o más partos & $4(5$ \\
& &
\end{tabular}

Tabla 2.

Distribución porcentual zona corporal con molestias músculo-esqueléticas (dolor) en los últimos 7 días

$\begin{array}{lcc}\text { Zona corporal con } & \begin{array}{c}\text { Frecuencia } \\ \text { Absoluta }\end{array} & \begin{array}{c}\text { Distribución } \\ \text { porcentual }\end{array} \\ \text { dolor últimos 7 dias } & 15 & 18,8 \% \\ \text { Cuello } & 8 & 10,0 \% \\ \text { Hombro derecho } & 8 & 10,0 \% \\ \text { Hombro izquierdo } & 4 & 5,0 \% \\ \text { Codo / antebrazo derecho } & 2 & 2,5 \% \\ \text { Codo / antebrazo izquierdo } & 13 & 16,3 \% \\ \text { Mano / muñeca derecha } & 5 & 6,3 \% \\ \text { Mano / muñeca izquierda } & 20 & 25,0 \% \%^{*} \\ \text { Espalda alta } & 62 & 77,5 \% \%^{*} \\ \text { Espalda baja } & 34 & 42,5 \% \%^{*} \\ \text { Cadera / nalgas / muslos } & 12 & 15,0 \% \\ \text { Rodillas } & 21 & 26,3 \% \%^{*} \\ \text { Tobillos / pies } & & \end{array}$

${ }^{*}$ Molestias músculo-esqueléticas.

\section{Gráfico 1.}

Presencia de los principales factores físicos en los puestos de trabajo, referidos por las embarazadas.

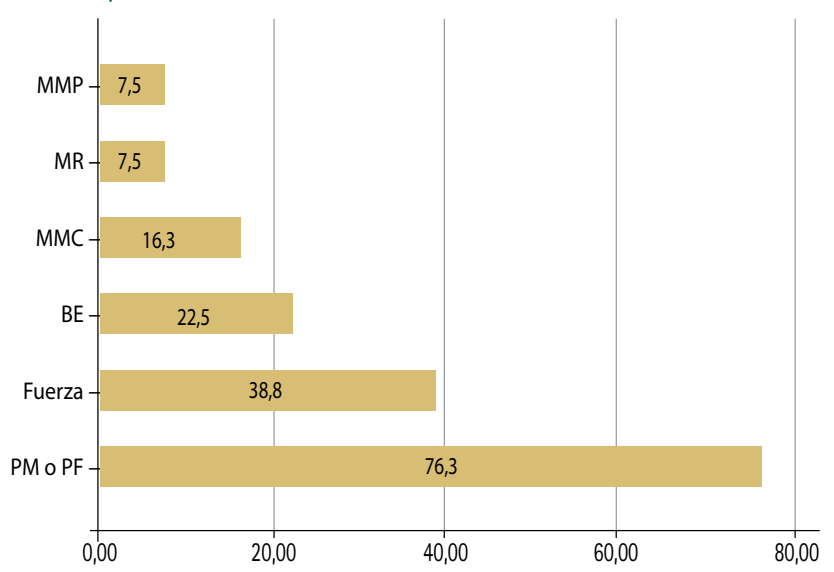

MMP: Manejo Manual de Pacientes; MR: Movimiento Repetitivo;

MMC: Manejo Manual de Carga; BE: Carga Bioenergética; Fuerza: Uso de fuerza; PM o PF: Postura Mantenida o Postura Forzada.

En el Gráfico 1 se describe la prevalencia de los factores físicobiomecánicos y en el Gráfico 2 las dificultades referidas por las mujeres embarazadas. De estos, destaca que las posturas mantenidas o forzadas fueron el factor más prevalente, con un 76,3\% de presentación, seguido de fuerza (38,8\%). Es importante destacar además que un 16,3\% refirió continuar realizando manejo manual de carga, y un 7,5\% manejo manual de paciente, esto aun estando embarazada. Por otro lado, la presencia de dificultades en relación a la tarea laboral o actividad de trabajo fueron también comúnmente percibidas, siendo las más frecuentes menor agilidad $(81,3 \%)$, fatiga $(76,3 \%)$ e incomodidad en la tarea (55\%).

La carga global de trabajo, según el NASA-TLX es descrita en la Tabla 3. Se destaca el elevado porcentaje de mujeres embarazadas que percibieron una carga de trabajo con riesgo alto $(61,3 \%)$, seguido de riesgo moderado (35\%) y riesgo leve (3,75\%). En cuanto al análisis por dimensiones especificas del cuestionario NASA-TLX, las dimensiones que presentaron una correlación estadísticamente significativa con la puntuación ponderada total fueron la dimensión temporal, dimensión esfuerzo y dimensión rendimiento. Se encontró correlación significativa en cuanto a la cantidad de dificultades y las "dimensiones esfuerzo" (p 0,014) y “dimensión frustración” (p 0,024) y la puntuación ponderada total 


\section{Gráfico 2.}

Presencia de dificultades en los puestos de trabajo, referido por las embarazadas en porcentaje.

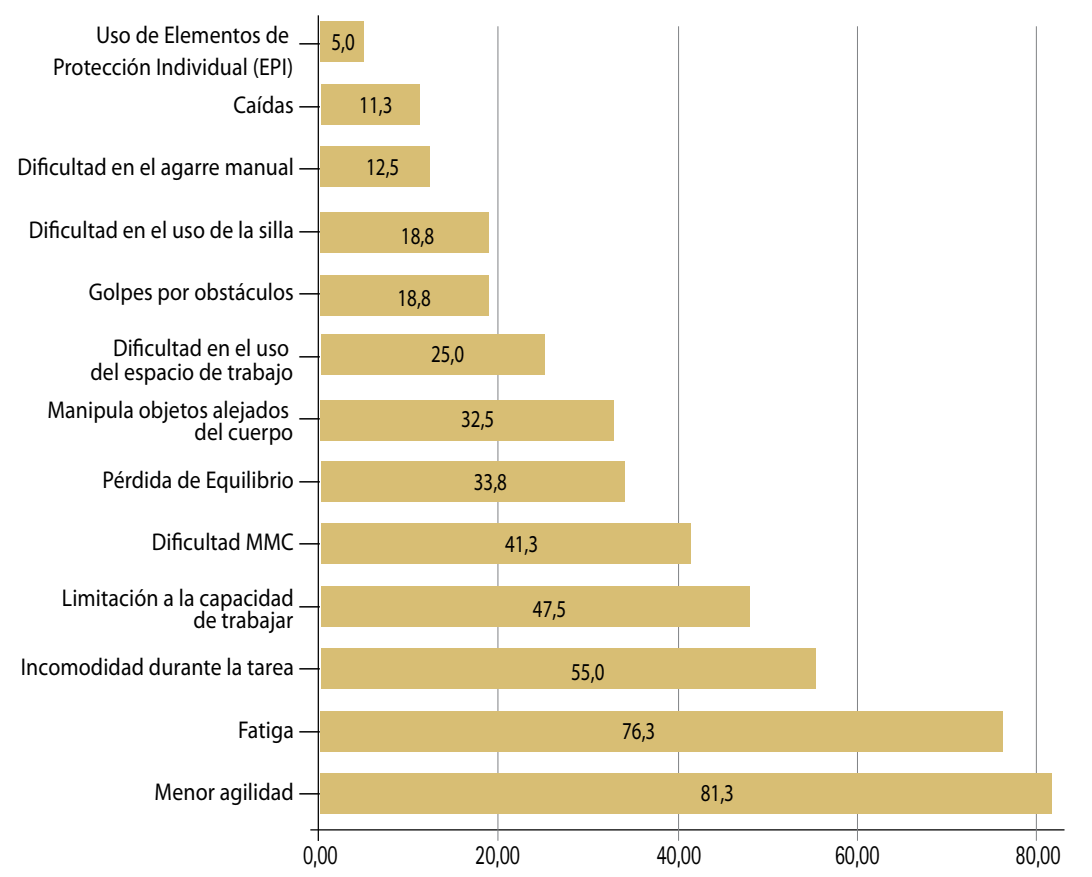

Segundo, en relación a los factores físico-biomecánicos, las posturas mantenidas o forzadas fueron el factor más prevalente, con un 76,3\% de presentación, dando cuenta de las características del puesto de trabajo; siendo necesario de controlar para evitar el riesgo de molestias músculo-esqueléticas en las embarazadas, especialmente en zonas lumbares, ya que se ha identificado al ejercicio físico y la movilidad como factores protectores ante el desarrollo de dolor lumbar según estudio realizado por Pennick, 2013. Además, es importante destacar que un 16,3\% refirió continuar realizando manejo manual de carga, y un 7,5\% manejo manual de paciente, esto aun estando embarazada. Este punto es crítico, ya que según la normativa nacional que regula el peso máximo de carga humana, la Ley 20.001 (2005), en su artículo 211-I, establece: "Se prohíben operaciones de carga y descarga manual para mujeres embarazadas" basado en el fundamento científico que asocia este factor físico con desenlaces desfavorables en el embarazo. ${ }^{26,28}$

Tercero, se identificó que la percepción de molestias músculo-esqueléticas son altamente frecuentes, con una prevalencia de $90 \%$ para la población total, dentro de lo cual las zonas corporales que presentaban mayor prevalencia fueron espalda baja (77,5\%), cadera/nalgas/muslos (42,5\%), tobillos/pies $(26,3 \%)$ y espalda alta (25\%). La percepción de MME en mujeres embarazadas son comunes tanto a nivel nacional como a nivel mundial. ${ }^{15-17}$ En un estudio realizado en una muestra de 30 mujeres embarazadas, las que se encontraban laboralmente activas presentaban más del 50\% de presencia de percepción de molestias músculo-esqueléticas. ${ }^{30}$

Se estima que afecta a la mitad de las mujeres embarazadas, de las cuales el 25\% experimenta dolor importante y el 8\% presenta severa discapacidad asociada. ${ }^{18} \mathrm{Si}$ bien se ha planteado que el estado nutricional, comprendido desde el Índice de Masa Corporal (IMC), se relaciona con la presencia de molestias músculo-esqueléticas, una reciente revisión demostró que la estatura y peso materno es un factor de riesgo de dolor lumbar con baja evidencia científica como causal de esta sintomatología. ${ }^{13}$ Esto se condice con los resultados obtenidos en este estudio, ya que no se encontró relación estadísticamente significativa entre las molestias músculo-esqueléticas e IMC en mujeres embarazadas.

Cuarto, la presencia de dificultades percibidas por las embarazadas en relación a la tarea laboral o actividad de trabajo que realiza, de las 13 dificultades, se observa que las que presentan mayor frecuencia relativa son: 1) percepción de menor agilidad $(81,3 \%)$, percepción de fatiga $(76,3 \%)$ e incomodidad en la tarea (55\%); cabe destacar que los cambios físicos y fisiológicos que presentan las embarazadas durante su proceso de gestación pueden verse reflejados de esta manera, siendo un aspecto relevante para considerar en la prevención de trastornos músculoesqueléticos en la mujeres trabajadoras que se encuentran en situación de embarazo.

Es necesario contextualizar estos resultados con las características de la muestra. Del total de la muestra evaluada, el 53,8\% de las embarazadas pertenecen al segundo trimestre de gestación y en cuanto al rubro productivo, un 95\% trabaja en el sector 
terciario, principalmente en puestos del sector servicio (comercio, enseñanza y servicios sociales entre otros), lo cual es concordante con la literatura, ya que la mayoría de las mujeres ocupadas de Chile y Latinoamérica trabajan en el sector servicio. ${ }^{2}$ Respecto a la condición de salud, reflejado en la evaluación del índice de masa corporal (IMC), para la población de mujeres embarazadas, según un estudio que se realizó en el año 2012, el 50\% de ellas presentaban algún grado de obesidad o sobrepeso ${ }^{15}$; este alto porcentaje se condice con nuestro estudio, ya que el 62,6\% de las embarazadas de la muestra presenta algún grado de obesidad o sobrepeso.

$\mathrm{Al}$ igual como se describe en este estudio, la existencia de molestias músculo-esqueléticas da cuenta de un proceso avanzado dentro de la progresión de desórdenes músculo-esqueléticos ${ }^{36-38}$, por lo que la efectividad de las actividades de prevención se ve reducida y se resalta la necesidad de prevención de la salud músculo-esquelética a través de la percepción de disconfort, como primer estadio posterior a la carga física a la cual se encuentra expuesta la trabajadora, además del propio estado de su embarazo. El desarrollo de estudios que permitan profundizar estrategias de prevención o control de los factores de riesgo, especialmente en los primeros dos trimestres de gestación, entre los diversos sectores productivos y un tamaño muestral mayor, será crítico.

Finalmente, se ha identificado una alta presencia de factores de riesgo ergonómicos de mujeres embarazadas, junto a niveles de carga mental entre moderada y alta. A su vez, la presencia de relaciones disergonómicas o dificultades se encuentran principalmente relacionadas con la agilidad, fatiga y comodidad. Es necesario establecer un entorno y una organización laboral que asegure comodidad y bienestar de la mujer en el puesto de trabajo junto a apoyo de medidas de autocuidado.

Es necesario realizar nuevos estudios en las mujeres embarazadas, considerando los distintos trimestres de gestación, que identifique la progresión de estos cambios a lo largo del embarazo, para detectar variables específicas que puedan influir en su relación con el entorno laboral según trimestre de gestación, considerando otros sectores productivos que no se encuentren feminizados, para detectar los factores de riesgos ergonómicos presentes y poder sectorizar las medidas preventivas. Además, es necesario explorar otros aspectos de la mujer en situación de embarazo, como considerar la calidad de vida relacionada a la salud de las embarazadas en el contexto de las actividades de la vida diaria, para analizar otros aspectos que indirectamente podrían influir en su ámbito laboral.

\section{Agradecimientos}

Queremos agradecer a la Fundación Científica y Tecnológica de la Asociación Chilena de Seguridad (FUCYT), al Departamento de Obstetricia y Ginecología-Unidad Materno-fetal del Hospital Clínico de la Universidad de Chile ( HCUCH). Además de las matronas, secretarias y personal técnico del HCUCH, a la Klga. Paz Jorquera y Klga. Victoria Villalobos, y principalmente a las embarazadas que participaron voluntariamente en este proyecto. 
1. International Labour Organization (ILO). World employment and social outlook: trends 2015. Geneva: ILO; 2015.

2. Instituto Nacional de Estadistica. Mujeres en Chile y mercado del trabajo: Participación laboral femenina y brechas salariales. Santiago: INE; 2015.

3. Instituto Nacional de Estadística. Estadísticas Vitales 2013. Santiago: Gobierno de Chile; 2013.

4. Messing K. La sante des travailleuses. La science est-elle aveugle?. Toulouse; Octarès Editions; 2000.

5. Ministerio de Salud (MINSAL). Encuesta nacional de calidad de vida y salud. Informe institucional-ENCAVI. Santiago: Gobierno de Chile; 2007.

6. Rodríguez $\mathrm{C}_{1}$ Mondelo $\mathrm{P}_{1}$ Cerda $\mathrm{E}$. Análisis de variables para la construcción de un modelo conceptual de tiempo de adaptación del puesto de trabajo según evolución del desarrollo gestacional [tesis doctoral]. Barcelona: Universitat Politècnica de Catalunya; 2016.

7. Marcinkiewicz A, Wezyk A, Muszynski P, Polanska K, Makowiec-Dabrowska T, Wiszniewska $M_{1}$ et al. Good practice in occupational health services; The influence of hazardous conditions and nuisance coexisting in the work environment and at home on the course and outcome of pregnancy. Med Pr. 2015; 66(5):713-24

8. MacDonald LA, Waters TR, Napolitano PG, Goddard DE, Ryan MA, Nielsen P, et al. Clinical guidelines for occupational lifting in pregnancy: evidence summary and provisional recommendations. Am J Obst Gynecol. 2013; 209(2):80-8.

9. Lile J, Perkins J, Hammer RL, Loubert PV. Diagnostic and management strategies for pregnant women with back pain. JAAPA. 2003; 16(12):31-36, 38-39, 44.

10. Morrissey SJ. Workplace design recommendations for the pregnant worker. Int $J$ Ind Ergonom. 1998; 21(5):383-395.

11. Fagarasanu $M$, Kumar $S$. Carpal tunnel syndrome due to keyboarding and mouse tasks: a review. Int J Ind Ergonom. 2003; 31(2):119-136.

12. Brown $A$, Johnston R. Maternal experience of musculoskeletal pain during pregnancy and birth outcomes: significance of lower back and pelvic pain. Midwifery. 2013; 29(12):1346-1351

13. Munjin $M$, llabaca $F$, Rojas J. Dolor lumbar relacionado al embarazo. Rev Chi Obstet Ginecol. 2007; 72(4):258-65.

14. Zhou K, West HM, Zhang J, Xu L, Li W. Interventions for leg cramps in pregnancy. Cochrane Database of Systematic Reviews [on line]. 2015; (8) [cited Jan 2017]. Available from: http://www.cochrane.org/CD010655/PREG_interventions-leg-cramps-during-pregnancy

15. Rodriguez C, Gutiérrez L, Cortés M, Cerda E. Estudio de la Carga Global de Trabajo y Percepción de Molestias Músculo-Esqueléticas en Embarazadas Atendidas en la Unidad de Maternidad del Hospital Clínico de la Universidad de Chile. Cienc Trab. 2011; 13(39):53-56.

16. Sabino J, Grauer JN. Pregnancy and low back pain. Curr Rev Musculoskelet Med. 2008; 1(2):137-141.

17. Paul JA, Frings-Dresen MH, Salle HJ, Rozendal RH. Pregnant women and working surface height and working surface areas for standing manual work. Appl Ergon. 1995; 26(2):129-33.

18. Dumas GA, Upjohn TR, Delisle A, Charpentier K, Leger A, Plamondon A, et al Posture and muscle activity of pregnant women during computer work and effect of an ergonomic desk board attachment. Int J Ind Ergonom. 2009; 39(2):313-25.

19. Ministerio de Empleo y Seguridad Social. Carga de trabajo y embarazo. Madrid: Gobierno de España; 1996.

20. Cheng CY, Pickler RH. Perinatal stress, fatigue, depressive symptoms, and immune modulation in late pregnancy and one month postpartum. ScientificWorldJournal. 2014; Jan 22; 2014:652630.

21. Marc I, Toureche N, Ernst E, Hodnett ED, Blanchet C, Dodin S, et al. Mindbody interventions during pregnancy for preventing or treating women's anxiety. Cochrane Database of Systematic Reviews [on line]. 2011; (7) [cited Jan 2017]. Available from: https://www.academia.edu/20016352/Mindbody_interventions_during_pregnancy_for_preventing_or_treating _ womens_anxiety

22. Kramer MS, McDonald SW. Aerobic exercise for women during pregnancy. Cochrane Database of Systematic Reviews [on line]. 2006; (3) [cited Jan 2017]. Available from: http://onlinelibrary.wiley.com/doi/10.1002/14651858. CD000180.pub2/abstract.

23. Mogren IM. Previous physical activity decreases the risk of low back pain and pelvic pain during pregnancy. Scand J Public Health. 2005; 33(4):300306.

24. Rivilis I, Van Eerd D, Cullen K, Cole DC, Irvin E, Tyson J, et al. Effectiveness of participatory ergonomic interventions on health outcomes: A systematic review. Appl Ergon. 2008; 39(3):342-358.

25. Bastiaanssen JM, de Bie RA, Bastiaenen $\mathrm{CH}$, Essed GG, van den Brandt PA. A historical perspective on pregnancy-related low back and/or pelvic girdle pain. Eur J Obstet Gynecol Reprod Biol. 2005; 120(1):3-14.

26. Bonzini M, Coggon D, Palmer KT. Risk of prematurity, low birthweight and preeclampsia in relation to working hours and physical activities: a systematic review. Occup Environ Med. 2007; 64(4):228-243.

27. Haas JS, Jackson RA, Fuentes-Afflick E, Stewart AL, Dean ML, Brawarsky $P$, et al. Changes in the Health Status of Women During and After Pregnancy. J Gen Int Med. 2005; 20(1):45-51.

28. Cheng PL, Pantel M, Smith JT, Dumas GA, Leger AB, Plamondon A, et al. Back pain of working pregnant women: Identification of associated occupational factors. Appl Ergon. 2009; 40(3):419-23.

29. Atalah, E. Epidemiología de la Obesidad en Chile. Rev Med Clin Las Condes. 2012; 23(2):117-123.

30. Cortés, M, Gutiérrez, I y Rodríguez, C. Estudio de la carga global de trabajo y percepción de molestias músculo-esqueléticas en embarazadas atendidas en la unidad de maternidad del Hospital Clínico de la Universidad de Chile [tesis]. Santiago: Universidad de Chile; 2011.

31. Mehta RK, Agnew MJ. Subjective Evaluation of Physical and Mental Workload Interactions across Different Muscle Groups. J Occup Environ Hyg. 2015; 12(1):62-68.

32. Córdova V, Cerda E, Rodríguez C, Díaz C, Marchetti N, Behn C et al. Guía Técnica para la Evaluación del Trabajo Pesado. Santiago: Superintendencia de Pensiones-Universidad de Chile; 2010.

33. Martínez $M$, Alvarado R. Validación del Cuestionario Nórdico Estandarizado para la población trabajadora chilena [tesis Magister]. Santiago: Universidad de Chile; 2012.

34. Instituto de Biomecánica de Valencia (IBV). Ergomater/IBV: Requisitos ergonómicos para la protección de la maternidad en tareas con carga física [en línea]. Valencia: IBV; 2007 [citado ene 2017]. Disponible en: https:// www.uclm.es/servicios/prevencion/documentacion/maternidad/ibv\%20 requisitos $\% 20$ erg $\% 20$ maternidad $\% 20$ con $\% 20$ carga $\% 20$ foc3\%adsica_ ergomater[1].pdf.

35. Hansen $\mathrm{ML}$, Thulstrup $\mathrm{AM}$, Juhl $\mathrm{M}$, Kristensen JK, Ramlau-Hansen $\mathrm{CH}$. Predictorsof sickness absence in pregnancy: a Danish cohort study. Scand J Work Environ Health. 2015; 41(2):184-193.

36. Bastiaanssen J, Bie R de, Bastiaenen $C$, Heuts A, Kroese M, Essed G, van den Brandt P. Etiology and prognosis of pregnancy-related pelvic girdle pain: Design of a longitudinal study. BMC Public Health. 2005; 3(5):1.

37. Bergström $C$, Persson M, Mogren I. Pregnancy-related low back pain and pelvic girdle pain approximately 14 months after pregnancy - pain status, self-rated health and family situation. BMC Pregnancy Childbirth. 2014; 14:48.

38. Ferguson SA, Marras WS. A literature review of low back disorder surveillance measures and risk factors. Clin Biomech (Bristol).1997; 12(4):211-226. 